\title{
A Type-2 Fuzzy Logic Based System for Augmented Reality Visualisation of Georeferenced Data
}

\author{
Anasol Peña-Rios, Hani Hagras, Michael Gardner \\ The Intelligent Environments Research Group \\ School of Computer Science and Electronic Engineering, \\ University of Essex, Wivenhoe Park, \\ Colchester, UK.
}

\author{
Gilbert Owusu \\ Business Modelling and Operational Transformation \\ Practice, British Telecom, Adastral Park, Martlesham \\ Heath, Ipswich, UK
}

\begin{abstract}
Planning of infrastructure's provision and maintenance tasks is commonly done in a planning office using paper maps and desktop applications. However, any infrastructure plan has to be verified on location before being submitted to the responsible authorities. This task is usually accomplished by taking paper maps to the field and annotating them on site, or in the best case, using two-dimensional (2D) maps on mobile devices. Augmented reality (AR) can provide enhanced experiences of real-world situations by overlaying key information and three-dimensional (3D) visualizations when needed, thus supporting decision-making processes. AR could support land surveyors and mobile planners with a graphical overlay of the planned changes, highlighting relevant information and assets in their field of view. This paper presents an AR application, which uses interval type-2 fuzzy logic mechanisms to visualise immersive 3D georeferenced data; supporting planning and designing of infrastructure by directly modifying data to incorporate required changes, without the need of any postprocessing. Immersive visual feedback is provided via a head mounted display (HMD), enhancing user's 3D spatial perception of georeferenced data.
\end{abstract}

Keywords-fuzzy logic, type-2 fuzzy logic systems, augmented reality, geographical information system.

\section{INTRODUCTION}

Land surveyors collect data to determine terrestrial or three-dimensional (3D) positions of points, boundaries and locations of particular assets defining legal boundaries used in multiple scenarios including transport, communications, real estate or mapping. In the telecommunications domain, land surveys help in determining true position of existing company assets; in identifying characteristics of the land; and in creating reference points and markers that will guide the installation of new infrastructure to provide the services required by the customers. Usually, land surveyors rely on equipment such as Global Positioning System (GPS) receivers, 3D scanners, and Geographic Information Systems (GIS) to increase efficiency, accuracy and productivity. These tools are used to determine the position of Points of Interest (POIs) by measuring angles and distances between them. The factors that can affect the accuracy of their observations are also measured. They then use this data to create vectors, bearings, coordinates,

This work was supported by Innovate UK, British Telecom and Essex University as part of the Knowledge Transfer Project [KTP9985]. elevations, areas, volumes, plans and maps. Normally, infrastructure provisioning and maintenance tasks are designed and planned using paper maps, which are plotted as needed and manually annotated on site if changes are needed.

GIS are computer based systems designed to support the capture, management, manipulation, analysis, modelling and display of spatially referenced data at different points in time [1]. They are useful for generating infrastructure visualizations, cartography and calculations to assists decision-makers with urban analysis and planning. They provide a mechanism for data integration, management and analysis, and generate concise reports on spatial environment using multiple interrelated information, such as, cadastre maps, altitudes, urban plans, land use, economic development information, demographic information and various statistical analyses. One of the main benefits of GIS is that they allow for the reuse of information since data can be collected one time and shared for multiple scenarios and calculations. For improved efficiency, paper plans are increasingly being replaced by portable devices taken to the field to have direct access to GIS.

However, the capturing of geo datasets uses different methods and equipment, adding an extra factor of uncertainty to geographic data. In particular, the use of fuzzy logic combined with GIS allows for the incorporation of imprecise and vague information, improving the capturing of expert knowledge from urban planners, land surveyors and cartographers, representing geographical information more accurately. In addition, current visualisation capabilities of GIS are still unable to create a sense of place and realism sufficiently similar to that of the real world. Hence, there is a need for interactive visualisation systems, able to combine georeference data with a three-dimensional (3D) immersion system that uses real world elements to overcome several of the problems associated with current systems. Immersive visualisations would enable mobile planners and field surveyors to make an accurate judgment of a live situation from georeferenced data when they need to apply a mental transformation from map to reality. For example, when planning and designing infrastructural layouts.

In this paper, we present a type-2 fuzzy logic system (FLS) to support field surveyors with the capturing and updating of field data in real time using an Augmented Reality (AR) user 
interface. In this case, field surveyors determine the position of a POI (which could be the exact location of an existing telephone pole or other equipment, or the place where a new one should be installed). Using the AR application, surveyors can capture or modify POI's georeferenced metadata which is automatically converted from a two-dimensional (2D) model onto a three-dimensional (3D) view, calculating distances and terrain characteristics automatically. By means of an AR HeadMounted Device (HMD) the application overlays POIs information in the surveyors' field of view (FOV) allowing them to correct the data and carry out the design of new infrastructure in real-time. This facilitates faster responses with the aim of improving customer service. An overall view of the POIs in the area can be also visualised via a $2 \mathrm{D}$ map available in a companion mobile app.

The rest of this paper is structured as follows: Section II introduces a brief overview of the key concepts such as augmented reality, geographical information systems and fuzzy systems, and related applications. Section III presents the proposed type-2 fuzzy logic-based system for land surveying using augmented reality. Section IV presents the experiments and results while the conclusions and future work are presented in Section V.

\section{LITERATURE REVIEW}

\section{A. Geographical Information Systems}

A GIS is a computer-based system that provides at least four capabilities to handle georeferenced data [2] [3]:

- Data capture and preparation: Typically achieved using different techniques such as observation and manual measurement, using diverse measurement instruments and sensors, such as GPS receivers. Measurements are often split into horizontal and vertical components to simplify calculation, whilst GPS and astronomic measurements also include the measurement of a time component.

- Data management: Refers to storage and maintenance of information, which needs to be put into a digital format or computer-readable form to be used for further analysis.

- Data manipulation and analysis: Involves the calculation and analysis of georeferenced data through to the eventual production of visualisations. Positional data, also called spatial or georeferenced data, indicates where POIs are (or where they will be), which is in relation to geographic space, which we define as positional data relative to the Earth's surface [3] using a spatial referencing system (e.g. longitude, latitude).

- Data visualisation: Deals with formatting georeferenced data into a visualisation that communicates the result of data analysis in the best possible way. This is commonly presented as maps, which can be further combined with the dimensions of the graphically represented spatial data [3]. GIS maps are layered, with each layer consisting of information related to the area contained in the map, such as spatial objects (roads, rivers, lakes, etc.), population distribution, land division, etc.
Particularly, because GIS aims to solve problems that deal with $3 \mathrm{D}$ data, there is a need for new visualization techniques for other than just 2D maps in order to represent geographical reality. It is our belief that immersive technologies such as virtual reality (VR) and augmented reality (AR) can be used to improve GIS visualization and provide an effective alternative as we reported in [4], [5].

\section{B. Augmented Reality}

Augmented reality (AR) could be best envisioned as a broad concept, rather than a specific technology or set of technologies. It interactively combines the physical world with virtual elements in real time, where virtual elements usually appear three-dimensionally to users [6]. To do so, AR relies on a combination of technologies to identify specific objects or POIs; superimposing computer-generated content over a realworld environment. It relies on display technologies to show virtual elements, tracking technologies using computer vision or positioning sensors to identify POIs and objects, and interaction technologies to allow users to interact with virtual elements and overlaid information.

AR presents multiple possibilities for its inclusion in the workplace due to the possibility of overlaying key information and three-dimensional (3-D) visualizations when needed in real-world situations. It has been used in a number of application areas such as industrial [7], [8], [9], medical [10], military [11], and georeferenced data applications for agriculture [12], underground infrastructure visualization [7], and architecture [13], which demonstrate how these areas could benefit from augmentation. Moreover, capturing physical elements of a space, could give a better understanding of human activities [14].

Smart glasses are a combination of wearable devices and AR technologies [15]. The main advantage of using AR smart glasses is its hands-free capability, offering users greater flexibility. Current augmented reality HMD devices have a number of limitations for their use in outdoor activities, and most of them do not incorporate geolocation capabilities, such as GPS receivers or the capability to convert 2D georeferenced data into 3D spatial coordinates.

\section{Type-2 Fuzzy Systems}

Fuzzy logic attempts to mimic human thinking by emulating the approximate reasoning of humans by representing vague terms in a quantitative way, playing an important role in modelling and representing imprecise linguistic human concepts, using linguistic IF-THEN rules to model behaviour in a human-readable form [16], [17].

In classical set theory, a set has precisely defined boundaries, which means that an element has either full or no membership in the set (Boolean logic). Fuzzy logic extends classical set theory to calculate intermediate values between true and false, providing a smooth transition when encountering uncertainties; performing well despite the noise and imprecision in real-world settings. However, while type-1 (T1) fuzzy sets do well managing slight uncertainties (e.g., imprecision associated with sensors and actuators, or slight user-behaviour changes), type-2 (T2) fuzzy sets are designed to 
model and handle higher levels of uncertainty, such as those present in real-world environments [18], [19]. This is because T1 fuzzy sets use precise and crisp membership functions, whereas T2 fuzzy sets use fuzzy membership functions (i.e., the membership value for each element is a fuzzy set) instead of a crisp number - as in [0,1]. Research in [17] and [18] present examples of T2 fuzzy logic control and automation.

Fig. 1 illustrates a standard Interval T2 (IT2) fuzzy set, where the dotted lines represent the primary membership functions (MF) for each fuzzy set (T1) and the solid lines represent the lower $\underline{\mu}_{\tilde{A}}(x)$ and upper $\bar{\mu}_{\tilde{A}}(x)$ MFs of the T2 fuzzy set. To produce the corresponding T2 fuzzy sets, a bounded region was generated around the primary memberships, as a result of an uncertainty factor, to produce the Footprint of Uncertainty (FOU) of the system. Thus, T2 fuzzy sets provide two degrees of freedom via the FOU and the $3 \mathrm{D}$ nature of these sets [19].

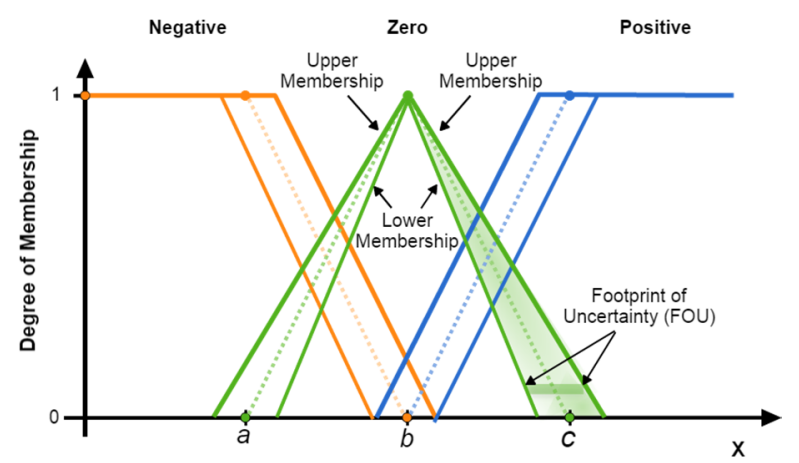

Fig. 1. An interval type-2 fuzzy set.

Fig. 2 shows the structure of a standard T2 fuzzy logic system [22], in which crisp inputs are first fuzzified, converting them to input T2 fuzzy sets. Then, the inference engine identifies the rules fired from a previously defined rule base, combining them to produce output T2 fuzzy sets. After that, the T2 fuzzy output sets are reduced, mapping them to T1 fuzzy sets (also known as type-reduced sets) by combining them and performing a centroid calculation. Finally, the typereduced sets are defuzzified (by taking the average of the typereduced set) to obtain crisp system [22].

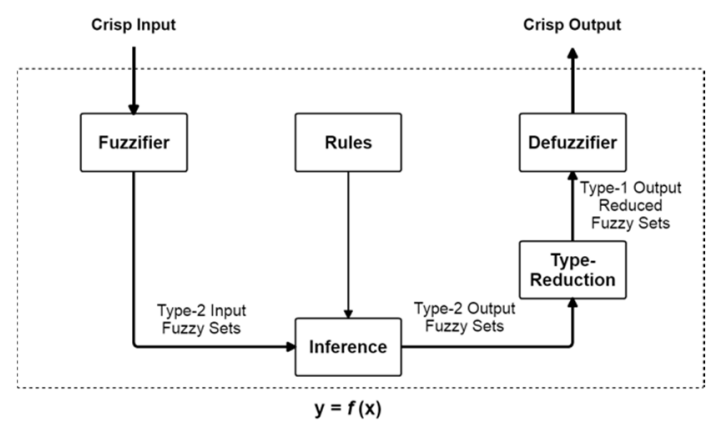

Fig. 2. Structure of a type-2 FLS [22].

Fuzzy logic has been used with GIS for fuzzy spatial analysis [23], [24], fuzzy reasoning [25], [26], [27], and the representation of fuzzy boundaries in maps [28] [1].

\section{The Proposed T1/T2 FuZZY LogIC BASED GIS-AR SYSTEM}

In this paper, we propose a novel application combining a GIS with a FLS which uses an AR headset to capture or update georeferenced POIs, allowing surveyors to work hands-free when validating and designing infrastructure developments whilst experiencing total immersion. Immersion can be defined as "a set of physical properties that give rise to presence" [29], whilst Presence is the sense of being in a place or environment [30], and it could be considered as the key concept that allows for the creation of a synthetic reality in terms of human experience rather than technological hardware. However, it is directly dependant on the user's perceptual feedback via the appropriate technology [29].

Our system uses a GPS receiver and a compass sensor in a mobile device to capture location and orientation of the user, sending this information to a server which compares the orientation with the head mounted device, and calculates the distances of $3 \mathrm{D}$ objects in relation with the user's position. The GPS is a global navigation system based on satellite information, owned and maintained by the United States government; and accessible to anyone with a GPS receiver [31]. It measures the user's 3D position providing worldwide coverage, however, it does not directly measure their orientation. GPS accuracy depends on multiple factors, including atmospheric effects, sky blockage, and receiver quality [31]. However, higher accuracy can be attainable by using GPS in combination with augmentation systems [6].

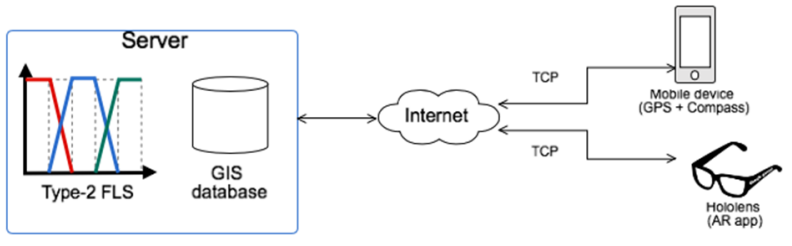

Fig. 3. Proposed System - Conceptual Model.

When users need to make an accurate judgment of a live situation from a map and a GPS location they need to apply a mental transformation from map to reality [7]. This process involves a number of factors, such as the user's familiarity with map scales, generalization and symbol language, which all have an effect in the accuracy of their viewpoint. Even experienced users may struggle if, for example, reference points are occluded [7]. Geospatial data cannot be directly visualized using AR, since it consists of a collection of $2 \mathrm{D}$ georeferenced points with abstract attributes or metadata (e.g. ID, object type, address, etc.). Our implementation uses a IT2 FLS to take geospatial data and translate it into 3D spatial data to be presented as a 3D object within a user's Field of View (FOV), taking his/her position as the initial reference point. In addition, we use a T1 FLS to determine a 3D object's behaviour and interaction capabilities. To do so, firstly the user needs to start the companion mobile app, which automatically sends the user's position and orientation in real-time to the server. Then, the server sends these values to the AR app in the HMD (as shown in Fig. 3). Once the communication has been established and the AR app is aware of the user's coordinates 
and orientation, the immersive AR app then asks the user to face North to establish an initial reference working area of $9 \mathrm{~m}^{2}$ $(3 \times 3 \mathrm{~m})$, placing the user in the middle (Fig. $4 \mathrm{a})$. From there it identifies any existing POIs within this area and calculates the distances with relation to the user using fuzzy classification. Therefore, if a POI is too far, it won't be shown in the user's FOV; if it is at medium distance it will be shown but the user cannot interact with it. Finally, if the object is close, the user has full access to its metadata and can interact with it. This behaviour was designed to avoid cluttering the user's FOV, since this could decrease their experience, overwhelming them, and potentially could even be dangerous, occluding the user's vision if the area has a profusion of POIs. Thus, when the user moves within the working area, he will only see and interact with the objects closer to their current position.

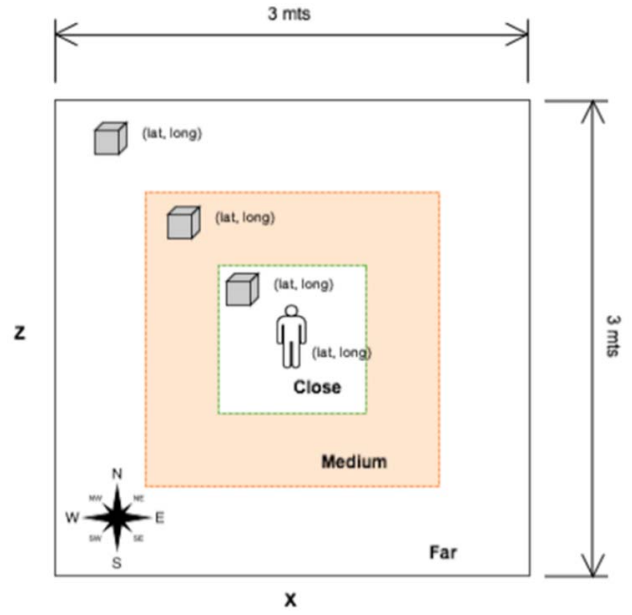

(a)

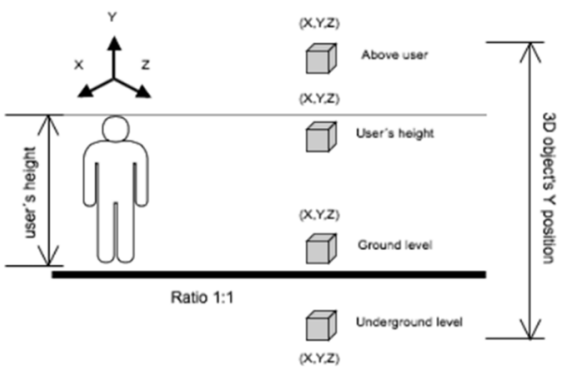

(b)

Fig. 4. System Implementation. a) User's working area. b) 3D object's $\mathrm{Y}$ position (with no terrain elevation).

Since previous HMDs outdoor evaluation studies have shown that text and colours could appear altered or washed out on the displays in daylight; an additional setting to adjust the AR app for outdoor use was considered. Previous studies [32], [33] recommend augmented elements to be $10-15 \%$ brighter than the background; this is in order to make them visible on a stereoscopic display. Hence, the daylight intensity level is used to adjust the brightness of virtual elements and $3 \mathrm{D}$ objects.

Finally, to transcode georeferenced data into 3D spatial coordinates $(\mathrm{X}, \mathrm{Y}, \mathrm{Z})$ for the $\mathrm{AR}$ scenes, we use an Interval Type-2 FLS (IT2FLS) considering the user's height, object's category, and terrain elevation. Fig. $4 \mathrm{~b}$ shows the different positions where a $3 \mathrm{D}$ object could be placed on the $\mathrm{Y}$ axis, depending on its classification (without considering terrain elevation or differences in users' height). For example, depending on the type, cables could be placed underground or above the user's head, thus they should be in the correct position in the AR scene to make the scene as realistic as possible. Address labels should appear at the user's eye level to be easily located. Poles and transducers have usually a standard regulated height, thus, they should be placed above the user's height.

\section{A. Implementation}

The system was implemented using Microsoft's HoloLens Development Edition (Fig. 5); a self-contained see-through AR headset, which runs Windows 10 with 64GB Flash, 2GB RAM, an Intel 32 bit architecture with TPM 2.0 support processor plus a custom-built Microsoft Holographic Processing Unit (HPU 1.0) [34]. Navigation along an area with a mobile AR GIS application could be cumbersome, due to the narrow field of view, plus the need to hold the device, leaving just one hand free. The use of Head-Mounted Devices (HDM), such as the HoloLens, offers greater flexibility by allowing users to work hands-free.
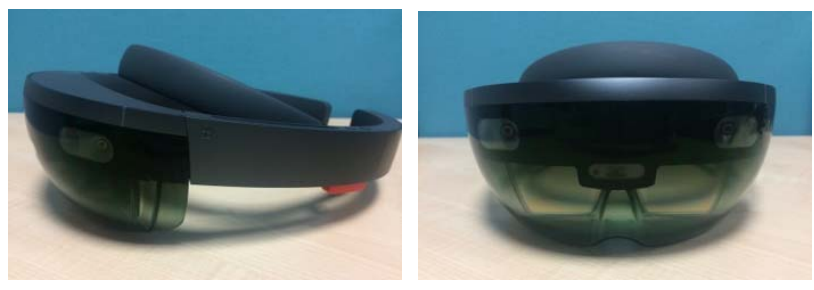

Fig. 5. Microsoft HoloLens.

Although the HoloLens includes indoor positioning sensors, access to them is restricted. In addition, they lack outdoor positioning devices such as GPS. Thus, for our experiment, we used values from the Inertial Measurement Unit (IMU) in a Microsoft Lumia 640 XL LTE mobile phone, with a quad core $1200 \mathrm{MHz}$ Qualcomm Snapdragon 400 processor, $8 \mathrm{~GB}$ of internal memory, 1 GB RAM, magnetometer and A-GPS, running on Windows 10 operating system [35].

Both, the companion mobile app and the immersive AR app were created using Microsoft Visual Studio 2015 and Unity ${ }^{1}$, a cross-platform game engine for creating interactive 3D content. Synchronisation between devices was achieved using SmartFox Server ${ }^{2}$, a middleware application used for large scale multiplayer games, massively multiplayer online games (MMO) and virtual communities.

\section{B. The T1 Fuzzy Logic System Design for Object's Visibility and Interaction}

We implemented a Type-1 FLS to determine each 3D object's behaviour and interaction capabilities, because the evaluated variables did not present high levels of uncertainty,

\footnotetext{
${ }^{1}$ Unity - www.unity.com

${ }^{2}$ Smart Fox Server - www.smartfoxserver.com
} 
therefore a type-2 implementation was not necessary. Fig. 6 shows an example of a user's position and some POIs from a $2 \mathrm{D}$ view (Shown in Fig. 6a), where the closest are green, the ones at medium distance are yellow and the farthest are red. Fig. 6b illustrates the first-person view using the AR HMD. Here, the user is able to see the closest POIs (plus its associated metadata), if any POI was classified by the system as close to the current's user position. (a)

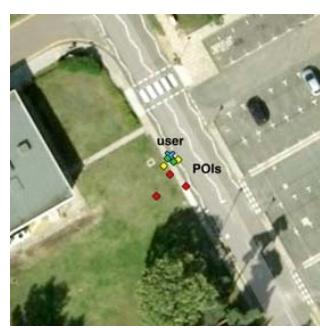

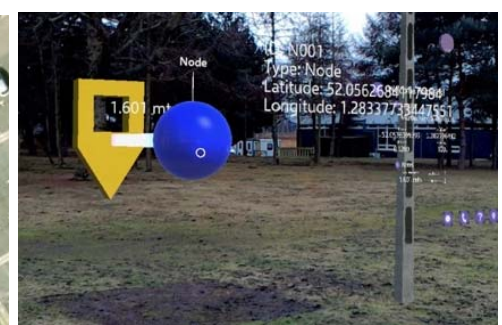

(b)
Fig. 6. T1 FLS Implementation. a) User's position and closest POIs. b) View from HMD.

Table I summarises the fuzzy rules to adjust an objects' visibility and interaction. Distances between POIs and user position were calculated using the haversine formula (1) that determines the great-circle distance between two points on a sphere given their longitudes and latitudes [36]. Once the distances are calculated, values are fuzzified using the membership function (MF) depicted in Fig. 7a.

$\operatorname{hav}\left(\frac{d}{r}\right)=\operatorname{hav}\left(\varphi_{2}-\varphi_{1}\right)+\cos \left(\varphi_{1}\right) \cos \left(\varphi_{2}\right) \operatorname{hav}\left(\lambda_{2}-\lambda_{1}\right)$

Where

- $\quad h a v$ is the haversine function (2)

$$
\operatorname{hav}(\theta)=\sin ^{2}\left(\frac{\theta}{2}\right)=\frac{1-\cos (\theta)}{2}
$$

- $\quad d$ is the distance between the two points (along a great circle of the sphere).

- $\quad r$ is the radius of the sphere.

- $\varphi_{2}, \varphi_{1}$ are latitude of point 1 and latitude of point 2 in radians.

- $\quad \lambda_{2}, \lambda_{1}$ are longitude of point 1 and longitude of point 2 in radians.

Daylight intensity is manually defined by the user after login, and it can be modified afterwards using a settings option. Daylight intensity MF (Fig. 7b) was based on the Common Light Levels Outdoors from Natural Sources classification proposed by the US National Optical Astronomy Observatory [37]. Using the product t-norm to represent the AND logical connective, the system calculates the firing strengths of each rule to decide whether a rule will be fired in response to a specific input. When the inference engine returns a result for brightness and opacity values. This result is converted by the defuzzifier into a crisp number using height defuzzification. The brightness level (whose fuzzy sets are depicted in Fig. 7c) refers to colour saturation, understanding saturation as "colourfulness of a stimulus relative to its own brightness" [38]. The opacity level (whose fuzzy sets are shown in Fig. 7d) describes the transparency level, where 1 is not transparent at all, 0.5 is $50 \%$ see-through, and 0 is completely transparent.

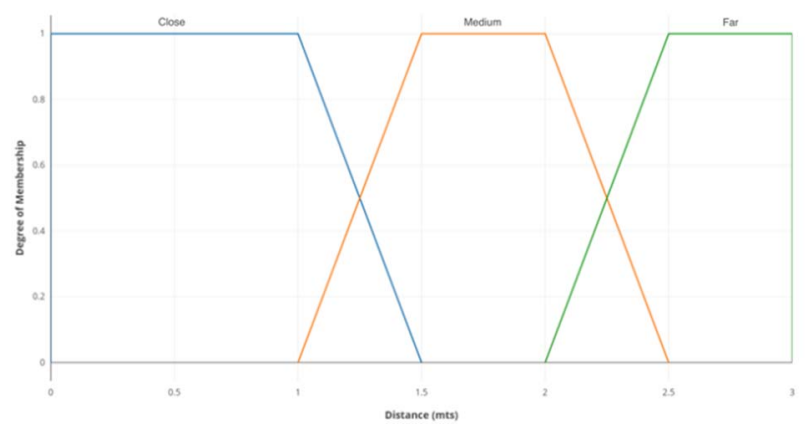

(a)

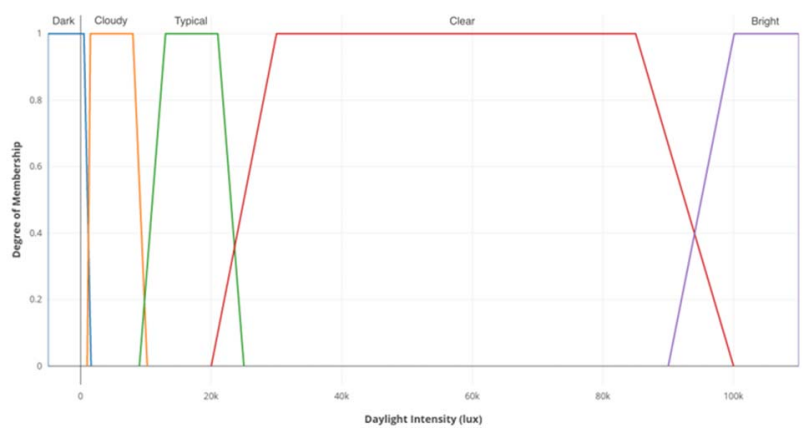

(b)

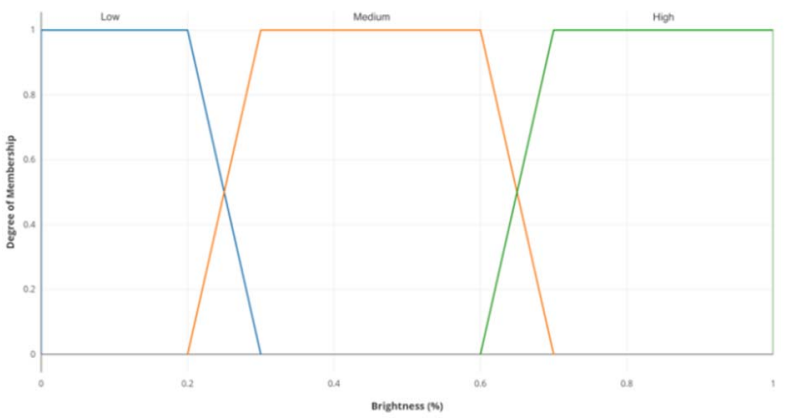

(c)

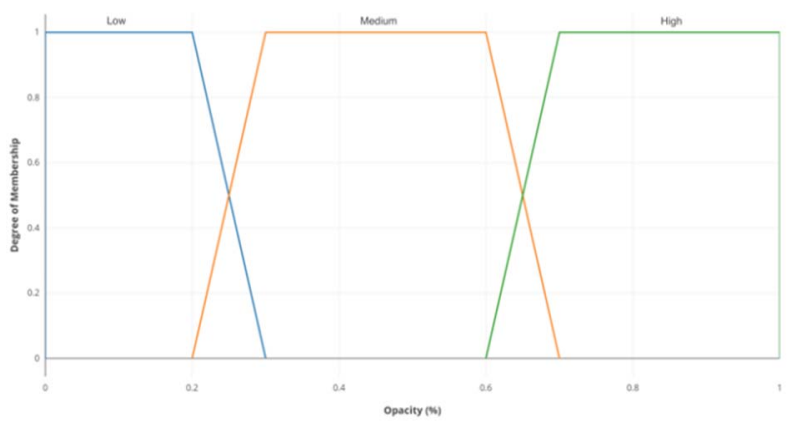

(d)

Fig. 7. Type-1 Membership Functions. Antecedents: a) Distance. b) Daylight Intensity. Consequents: c) Brightness. d) Opacity.

TABLE I. Rule BASE OF THE Proposed TyPe-1 FLS 


\begin{tabular}{|c|c|c|c|c|}
\hline \multirow{2}{*}{ Rule } & \multicolumn{2}{|c|}{ Antecedents } & \multicolumn{2}{c|}{ Consequents } \\
\cline { 2 - 5 } & D Distance & Daylight Intensity & Opacity & Brightness \\
\hline$R^{1}$ & Close & Dark & High & High \\
\hline$R^{2}$ & Close & Cloudy & High & High \\
\hline$R^{3}$ & Close & Typical & Medium & High \\
\hline$R^{4}$ & Close & Clear & Medium & High \\
\hline$R^{5}$ & Close & Bright & Medium & High \\
\hline$R^{6}$ & Medium & Dark & Medium & High \\
\hline$R^{7}$ & Medium & Cloudy & Medium & Medium \\
\hline$R^{8}$ & Medium & Typical & Medium & Medium \\
\hline$R^{9}$ & Medium & Clear & Medium & Medium \\
\hline$R^{10}$ & Medium & Bright & Medium & Medium \\
\hline$R^{11}$ & Far & Dark & Low & Medium \\
\hline$R^{12}$ & Far & Cloudy & Low & Medium \\
\hline$R^{13}$ & Far & Typical & Low & Low \\
\hline$R^{14}$ & Far & Clear & Low & Low \\
\hline$R^{15}$ & Far & Bright & Low & Low \\
\hline
\end{tabular}

object's type included in POIs metadata, as this determines possible spatial positions. Terrain's elevation (whose fuzzy sets are shown in Fig. 9c) was obtained using Google Maps API, which requests elevation data directly from DEM (Digital Elevation Model) databases [41]. Table II shows the rule base, which returns a 3D object's $Y$ position. $X$ and $Z$ positions are converted from user's coordinates given by the GPS, using the initial working area (shown in Fig. 4a), placing the user in the middle with coordinates $(0,0)$.

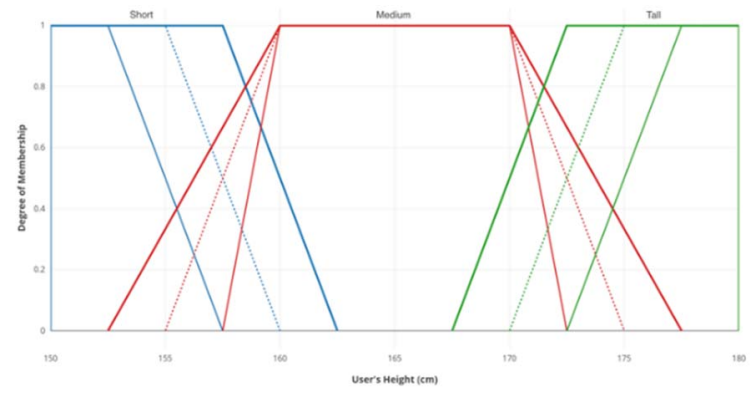

(a)

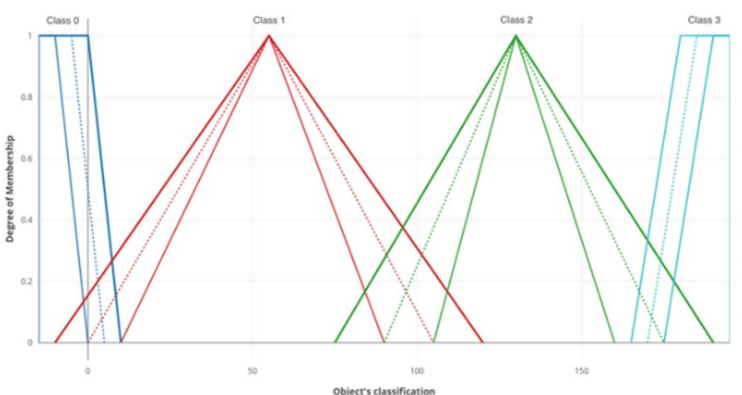

(b)

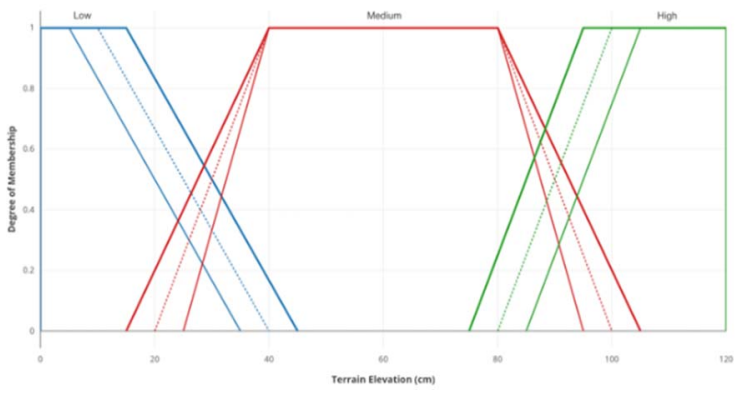

(c)

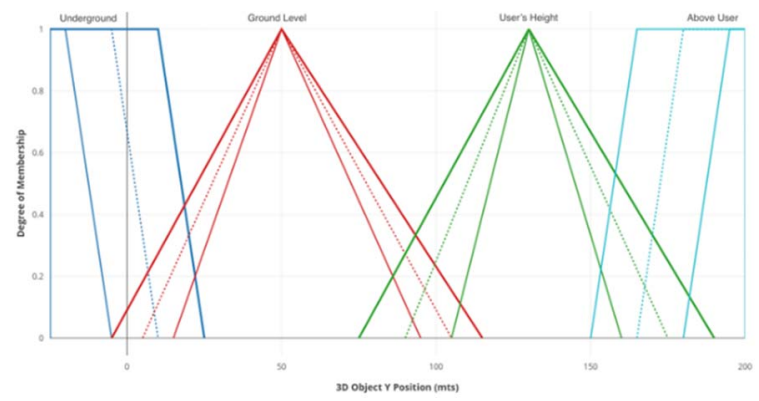

(d)

Fig. 9. Type-2 Membership Functions. Antecedents: a) User's Height. b) Object's Classification. c) Terrain's Elevation. Consequents: d) 3D Object Y Position. 
TABLE II. Rule BASE OF THE Proposed TyPe-2 FLS

\begin{tabular}{|c|c|c|c|c|}
\hline \multirow[b]{2}{*}{ Rule } & \multicolumn{3}{|c|}{ Antecedents } & \multirow{2}{*}{$\begin{array}{c}\text { Consequents } \\
3 D \text { Object Y } \\
\text { Position } \\
\end{array}$} \\
\hline & User's Height & $\begin{array}{c}\text { Object's } \\
\text { Classification }\end{array}$ & $\begin{array}{l}\text { Terrain's } \\
\text { Elevation } \\
\end{array}$ & \\
\hline$R^{1}$ & Short & Class 0 & Low & Underground \\
\hline$R^{2}$ & Short & Class 0 & Medium & Underground \\
\hline$R^{3}$ & Short & Class 0 & High & Ground level \\
\hline$R^{4}$ & Short & Class 1 & Low & Underground \\
\hline$R^{5}$ & Short & Class 1 & Medium & Ground level \\
\hline$R^{6}$ & Short & Class 1 & High & User's height \\
\hline$R^{7}$ & Short & Class 2 & Low & User's height \\
\hline$R^{8}$ & Short & Class 2 & Medium & User's height \\
\hline$R^{9}$ & Short & Class 2 & High & Above User \\
\hline$R^{10}$ & Short & Class 3 & Low & Above User \\
\hline$R^{11}$ & Short & Class 3 & Medium & Above User \\
\hline$R^{12}$ & Short & Class 3 & High & Above User \\
\hline$R^{13}$ & Medium & Class 0 & Low & Underground \\
\hline$R^{14}$ & Medium & Class 0 & Medium & Underground \\
\hline$R^{15}$ & Medium & Class 0 & High & Ground level \\
\hline$R^{16}$ & Medium & Class 1 & Low & Underground \\
\hline$R^{17}$ & Medium & Class 1 & Medium & Ground level \\
\hline$R^{18}$ & Medium & Class 1 & High & Ground level \\
\hline$R^{19}$ & Medium & Class 2 & Low & User's height \\
\hline$R^{20}$ & Medium & Class 2 & Medium & User's height \\
\hline$R^{21}$ & Medium & Class 2 & High & Above User \\
\hline$R^{22}$ & Medium & Class 3 & Low & Above User \\
\hline$R^{23}$ & Medium & Class 3 & Medium & Above User \\
\hline$R^{24}$ & Medium & Class 3 & High & Above User \\
\hline$R^{25}$ & Tall & Class 0 & Low & Underground \\
\hline$R^{26}$ & Tall & Class 0 & Medium & Underground \\
\hline$R^{27}$ & Tall & Class 0 & High & Underground \\
\hline$R^{28}$ & Tall & Class 1 & Low & Underground \\
\hline$R^{29}$ & Tall & Class 1 & Medium & Ground level \\
\hline$R^{30}$ & Tall & Class 1 & High & Ground level \\
\hline$R^{31}$ & Tall & Class 2 & Low & User's height \\
\hline$R^{32}$ & Tall & Class 2 & Medium & User's height \\
\hline$R^{33}$ & Tall & Class 2 & High & Above User \\
\hline$R^{34}$ & Tall & Class 3 & Low & Above User \\
\hline$R^{35}$ & Tall & Class 3 & Medium & Above User \\
\hline$R^{36}$ & Tall & Class 3 & High & Above User \\
\hline
\end{tabular}

To test the system, we carried out 300,000 simulations, in which, as a first step, an initial coordinate was defined (the user's position). Then our simulator generated random POIs coordinates within the working area of $9 \mathrm{~m}^{2}$. Each coordinate was then linked to terrain's elevation data and given an object classification randomly. Table III shows the average error and standard deviation obtained from comparing results between using a type-1 FLS and a type-2 FLS to generate in real time 3D object's Y coordinate, effectively converting 2D georeferenced data to 3D spatial coordinates. Here we can observe that the interval type-2 FLS (with uncertainty factor $\rho$ $=30 \%)$ produces lower average and standard deviation of errors (compared to the type-1 FLS) when generating spatial coordinates. FOU was adjusted empirically, selecting the one with the minor Standard Error of Mean (SEM) (fig. 10).

TABLE III. PERFORMANCE COMPARISON BETWEEN TYPE-1 FLS AND TYPE-2 FLS

\begin{tabular}{|c|c|c|c|}
\hline \multirow{2}{*}{ FLS } & \multicolumn{3}{|c|}{ Experimental Data Results } \\
\cline { 2 - 4 } & $\begin{array}{c}\text { Uncertainty } \\
\text { Factor }\end{array}$ & $\begin{array}{c}\text { Standard Error of } \\
\text { Mean (SEM) }\end{array}$ & $\begin{array}{c}\text { Standard } \\
\text { Deviation }\end{array}$ \\
\hline Type-1 & - & 0.871038869 & 61.5917491 \\
\hline Type-2 & $\mathbf{3 0} \%$ & $\mathbf{0 . 8 2 0 4 8 7 8 7 4}$ & $\mathbf{5 8 . 0 1 7 2 5 3 9 5}$ \\
\hline
\end{tabular}

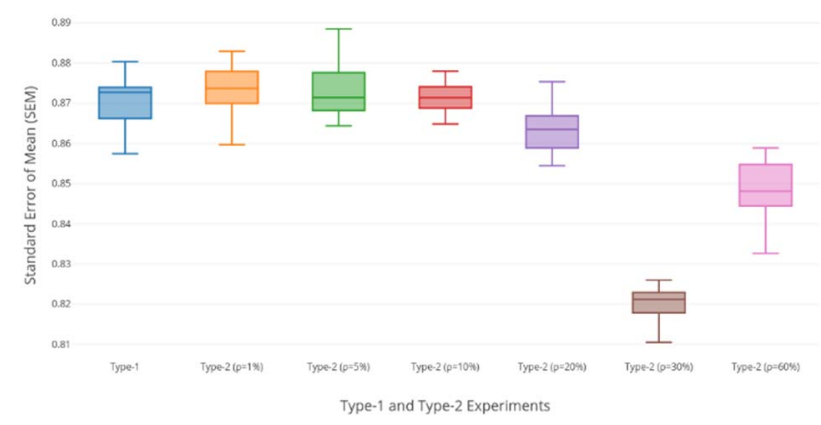

Fig. 10. Standard Error of Mean (SEM) obtained with different measures of uncertainty.

These results show an accuracy improvement (measured via RMSE) of $6 \%$ when using the type- 2 FLS, which could be due to the more precise values for the 3D object's coordinates. This verifies the type-2 FLS' ability to better handle the unseen uncertainties to be encountered in typical survey activities, producing better system output which better supports the allocation and visualisation of immersive $3 \mathrm{D}$ georeferenced data; supporting the planning and designing of infrastructure more accurately.

\section{CONCLUSiOnS ANd Future Work}

Augmented reality applications combined with fuzzy logic and geographic information systems (GIS) present an opportunity to enhance real-world scenarios. In this paper, we presented a novel application that combines fuzzy logic with augmented reality for the creation of immersive maps, allowing surveyors and planners to create and visualise POIs that can be visualised and manipulated in real time, converting 2D georeferenced points to 3D spatial positions. 
The proposed system showed an accuracy improvement of $6 \%$ when comparing our type- 1 to our type- 2 implementation. These results indicate that the type- 2 FLS was better in handling the short and long-term uncertainties.

In our future, on-going research, we intend to extend our implementation by adding collaborative capabilities, to allow the creation of POIs by remote users via a mobile/desktop app, whilst visualising them in real time using the AR application. Finally, we plan to perform a user evaluation of the proposed system, as well as continuing to develop the visual and auditory aids for the system to highlight specific areas or elements of interest.

\section{REFERENCES}

[1] A. Karabegovic, Z. Avdagic, and M. Ponjavic, "Applications of fuzzy logic in geographic information systems for multiple criteria decision making," 2006.

[2] S. Aronoff, "Geographic information systems: A management perspective," Geocarto Int., vol. 4, no. 4, p. 58, Dec. 1989.

[3] O. Huisman and R. A. De By, "Principles of geographic information systems," ITC Educ. Textb. Ser., vol. 1, 2009.

[4] A. Pena-Rios, H. Hagras, G. Owusu, and M. Gardner, “A Type-2 Fuzzy Logic Based System for Asset Geolocation within Augmented Reality Environments," in 2017 IEEE Int. Conf. on Fuzzy Systems (FUZZIEEE), 2017, pp. 1-6.

[5] A. Pena-Rios, H. Hagras, G. Owusu, and M. Gardner, "A Fuzzy Logic Based System for Geolocated Augmented Reality Field Service Support," in 2017 IEEE Int. Conf. on Fuzzy Systems (FUZZ-IEEE), 2017.

[6] R. Azuma and R. Azuma, "A survey of augmented reality," Presence Teleoperators Virtual Environ., vol. 6, no. 4, pp. 355-385, 1997.

[7] G. Schall et al., "Handheld Augmented Reality for underground infrastructure visualization," Pers. Ubiquitous Comput., vol. 13, no. 4, pp. 281-291, 2009.

[8] Airbus, "Augmented Reality for the Factory of the Future," 2015. [Online]. Available: https://goo.gl/tnCEg5. [Accessed: 10-Jan-2017].

[9] J. Zhu, S. K. Ong, and A. Y. C. Nee, "An authorable context-aware augmented reality system to assist the maintenance technicians," Int. J. Adv. Manuf. Technol., vol. 66, no. 9-12, pp. 1699-1714, 2013.

[10] T. Sielhorst, M. Feuerstein, and N. Navab, "Advanced medical displays: A literature review of augmented reality," IEEE/OSA J. Disp. Technol., vol. 4, no. 4, pp. 451-467, 2008.

[11] M. Livingston et al., "An Augmented reality system for military operations in urban terrain," in Interservice / Industry Training, Simulation, \& Education Conf. (I/ITSEC '02), 2002.

[12] G. R. King, W. Piekarski, and B. H. Thomas, "ARVino - Outdoor augmented reality visualisation of viticulture GIS data," Proc. - Fourth IEEE ACM Int. Symp. Symp. Mix. Augment. Reality, ISMAR 2005, vol. 2005, pp. 52-55, 2005.

[13] Y. Guo, Q. Du, Y. Luo, W. Zhang, and L. Xu, "Application of augmented reality gis in architecture," Int. Arch. Photogramm. Remote Sens. Spat. Inf. Sci., vol. XXXVII, pp. 331-336, 2008.

[14] S. Helal, J. W. Lee, S. Hossain, E. Kim, H. Hagras, and D. Cook, "Persim - Simulator for human activities in pervasive spaces," Proc. 2011 7th Int. Conf. Intell. Environ. IE 2011, pp. 192-199, 2011.

[15] P. A. Rauschnabel, A. Brem, and Y. K. Ro, "Augmented Reality Smart Glasses : Definition, Conceptual Insights , and Managerial Importance," Dearborn, 2015.

[16] H. Hagras, "Embedding Computational Intelligence in Pervasive Spaces," IEEE Pervasive Comput., vol. 6, no. 3, pp. 85-89, 2007.

[17] A. Kordon, Applying computational intelligence: how to create value. Springer Science \& Business Media, 2009.

[18] H. Hagras and C. Wagner, "Introduction to interval type-2 fuzzy logic controllers-towards better uncertainty handling in real world applications," IEEE Syst. Man Cybern. eNewsletter, vol. 27, Jun. 2009.
[19] H. Hagras, "Type-2 FLCs: A new generation of fuzzy controllers," IEEE Comput. Intell. Mag., vol. 2, no. 1, pp. 30-43, 2007.

[20] T. Kumbasar and H. Hagras, "A Self-Tuning zSlices-Based General Type-2 Fuzzy PI Controller," IEEE Trans. Fuzzy Syst., vol. 23, no. 4, pp. 991-1013, 2015.

[21] T. Kumbasar and H. Hagras, "Big Bang-Big Crunch optimization based interval type-2 fuzzy PID cascade controller design strategy," Inf. Sci. (Ny)., vol. 282, pp. 277-295, 2014.

[22] H. Hagras, "A Hierarchical Type-2 Fuzzy Logic Control Architecture for Autonomous Mobile Robots," IEEE Trans. Fuzzy Syst., vol. 12, no. 4, pp. 524-539, 2004.

[23] A. Zhu, B. Hudson, J. Burt, K. Lubich, and D. Simonson, "Soil Mapping Using GIS, Expert Knowledge, and Fuzzy Logic," Distribution, vol. 65, no. 5, pp. 1463-1472, 2001.

[24] G. Metternicht, "Assessing temporal and spatial changes of salinity using fuzzy logic, remote sensing and GIS. Foundations of an expert system," Ecol. Modell., vol. 144, no. 2, pp. 163-179, 2001.

[25] S. Dragicevic and D. J. Marceau, "An application of fuzzy logic reasoning for GIS temporal modeling of dynamic processes," Fuzzy sets Syst., vol. 113, no. 1, pp. 69-80, 2000.

[26] A. Gemitzi, C. Petalas, V. A. Tsihrintzis, and V. Pisinaras, "Assessment of groundwater vulnerability to pollution: A combination of GIS, fuzzy logic and decision making techniques," Environ. Geol., vol. 49, no. 5, pp. 653-673, 2006 .

[27] R. C. M. Nobre, O. C. Rotunno Filho, W. J. Mansur, M. M. M. Nobre, and C. A. N. Cosenza, "Groundwater vulnerability and risk mapping using GIS, modeling and a fuzzy logic tool," J. Contam. Hydrol., vol. 94, no. 3-4, pp. 277-292, 2007.

[28] B. Pradhan, "Use of GIS-based fuzzy logic relations and its cross application to produce landslide susceptibility maps in three test areas in Malaysia," Environ. Earth Sci., vol. 63, no. 2, pp. 329-349, 2011.

[29] W. A. Ijsselsteijn, "History of Telepresence," 3D Videocommunication Algorithms, Concepts Real-Time Syst. Hum. Centred Commun., pp. 521, Sep. 2006.

[30] M. Slater and S. Wilbur, "A Framework for Immersive Virtual Environments (FIVE): Speculations on the Role of Presence in Virtual Environments," Presence Teleoperators virtual Environ., vol. 6, no. 6, pp. 1-20, Dec. 1997.

[31] US National Coordination Office for Space-Based Positioning Navigation and Timing, "GPS Accuracy," 2017. [Online]. Available: https://goo.gl/Mjzrrk. [Accessed: 10-Jan-2017].

[32] S. D. Peterson, M. Axholt, and S. R. Ellis, "Label segregation by remapping stereoscopic depth in far-field augmented reality," Proc. - 7th IEEE Int. Symp. Mix. Augment. Real. 2008, ISMAR 2008, pp. 143-152, 2008.

[33] T. Pingel and K. Clarke, "Assessing the usability of a wearable computer system for outdoor pedestrian navigation," Autocarto ACSM, no. 805, 2005.

[34] Microsoft, "Hololens - Hardware details." [Online]. Available: https://developer.microsoft.com/en-us/windows/mixedreality/hololens_hardware_details. [Accessed: 24-Nov-2017].

[35] Microsoft, "Lumia 640 XL LTE Specifications - Microsoft - Global." [Online]. Available: https://goo.gl/vp1qbq. [Accessed: 10-Jan-2017].

[36] G. Van Brummelen, Heavenly mathematics: the forgotten art of spherical trigonometry. Princeton University Press, 2013.

[37] N. O. A. Observatory, "Recommended Light Levels (Illuminance) for Outdoor and Indoor Venues," 2011.

[38] M. D. Fairchild, Color appearance models. J. Wiley, 2005.

[39] J. M. Mendel, Uncertain rule-based fuzzy logic systems: introduction and new directions. Prentice Hall PTR Upper Saddle River, 2001.

[40] A. Moody, "Adult anthropometric measures, overweight and obesity," Office for National Statistics, 2012.

[41] Google, "Google Maps JavaScript API V3 Reference" [Online]. Available: https://goo.gl/zcye3y. [Accessed: 28-Jan-2018]. 